\title{
Frustration of the isotropic-columnar phase transition of colloidal hard platelets by a transient cubatic phase
}

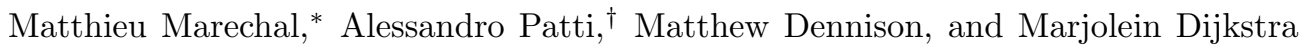 \\ Soft Condensed Matter, Debye Institute for NanoMaterials Science, \\ Utrecht University, Princetonplein 5, 3561 RT Utrecht, The Netherlands
}

(Dated: October 12, 2018)

\begin{abstract}
Using simulations and theory, we show that the cubatic phase is metastable for three model hard platelets. The locally favored structures of perpendicular particle stacks in the fluid prevent the formation of the columnar phase through geometric frustration resulting in vitrification. Also, we find a direct link between structure and dynamic heterogeneities in the cooperative rotation of particle stacks, which is crucial for the devitrification process. Finally, we show that the life time of the glassy cubatic phase can be tuned by surprisingly small differences in particle shape.
\end{abstract}

PACS numbers: 82.60.Nh,64.70.pv,82.70.Dd,64.70.M-

Nucleation is the process whereby a metastable phase transforms into a stable one, via the spontaneous formation of a cluster of the stable phase. According to classical nucleation theory, the free-energy barrier that separates the metastable phase from the stable state decreases with increasing supersaturation, and for quenches in the spinodal regime, the phase transformation proceeds via spinodal decomposition and coarsening. However, at sufficiently high supersaturations the motion of the particles can slow down so dramatically that the metastable state enters a glass regime.

Vitrification hampers the phase transformation as the particles cannot rearrange diffusively to form the stable phase. However, some glasses can evolve into the stable phase despite the arrested motion. The mechanism behind this so-called "devitrification" process is not well-understood, and neither the origin of the glass transition and its interplay with nucleation. An intriguing scenario based on geometrical frustration has been proposed, where the local order in the liquid phase is incompatible with the long-range order of the crystal phase [1. Hence, the formation of locally favored structures in the liquid, a concept proposed by Frank to explain dynamic arrest in glassy systems [2, prevents the crystallization. This scenario has been investigated using a two-dimensional lattice-free spin glass model, where the degree of frustration against crystallization can be tuned by an additional anisotropic potential that locally favors five-fold symmetry which is incompatible with the crystalline ground state of this model [1].

In this Letter, we investigate the interplay between nucleation, geometrical frustration, and devitrification in a simple (more realistic) 3D model system of colloidal hard platelets using computer simulations. The most common model systems for colloidal platelets are hard cut spheres (HCS), which consist of the middle section of thickness $L$ of a sphere of diameter $D$, and oblate hard spherocylinders (OHSC), comprising of a flat cylindrical core

\footnotetext{
* Currently at HHU, Düsseldorf, Germany

$\dagger$ Currently at IQAC-CSIC, Barcelona, Spain
}

with diameter $D$ and height $L$, and a toroidal rim with tube diameter $L$. OHSC are more rounded than HCS (see Fig. 1 1 ), and are therefore expected to better model the shape of colloidal disks, such as polymer-coated clay platelets 3 or charge-stabilised Gibbsite platelets [4. Interestingly, for $L / D=0.2$, the phase diagram of OHSC displays an isotropic-columnar (IC) phase transition [5], whereas a very peculiar cubatic phase was reported in between the isotropic and the columnar phase for HCS [6]. In this phase, the particles form small stacks of almost cube-like dimensions, which tend to align perpendicular to each other. Recently, it was shown that larger system sizes tend to destabilize the cubatic phase [7, 8. However, it remains an open question whether or not the cubatic phase is thermodynamically stable for colloidal hard platelets.

Here, we show that the cubatic phase of different model hard platelets is not stable, but should be considered as a transient phase in the IC phase transformation. In addition, we show that the degree of geometric frustration can be altered via subtle changes in the particle shape: the life time of the cubatic phase increases considerably a)

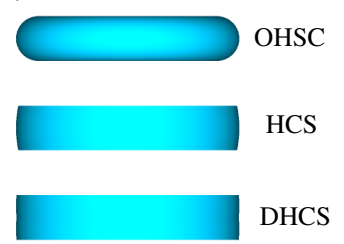

b)

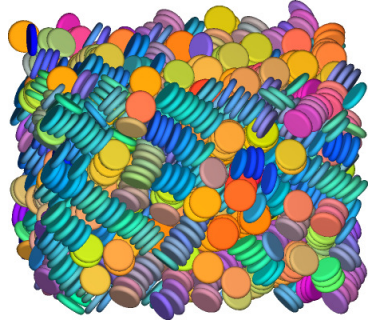

FIG. 1. (Color online) (a) Three model platelets: oblate hard spherocylinders (OHSC), hard cut spheres (HCS) and "double hard cut spheres" (DHCS). The volumes of the particles are given by $v_{\mathrm{OHSC}}=\pi L^{3} / 6+\pi^{2} \sigma L^{2} / 8+\pi \sigma^{2} L / 4$ with $\sigma=D-L$, $v_{\mathrm{HCS}}=\pi L\left(3 D^{2}-L^{2}\right) / 12$, and $v_{\mathrm{DHCS}}=\pi L\left(3 D^{2}-(L / 2)^{2}\right) / 12$ with $L$ and $D$ the (total) thickness and diameter of the particles, respectively. (b) A typical configuration of a cubatic phase of OHSC with $L / D=0.2$ and $P^{*}=11.25(\eta \simeq 0.57)$. Different colors denote different orientations. 

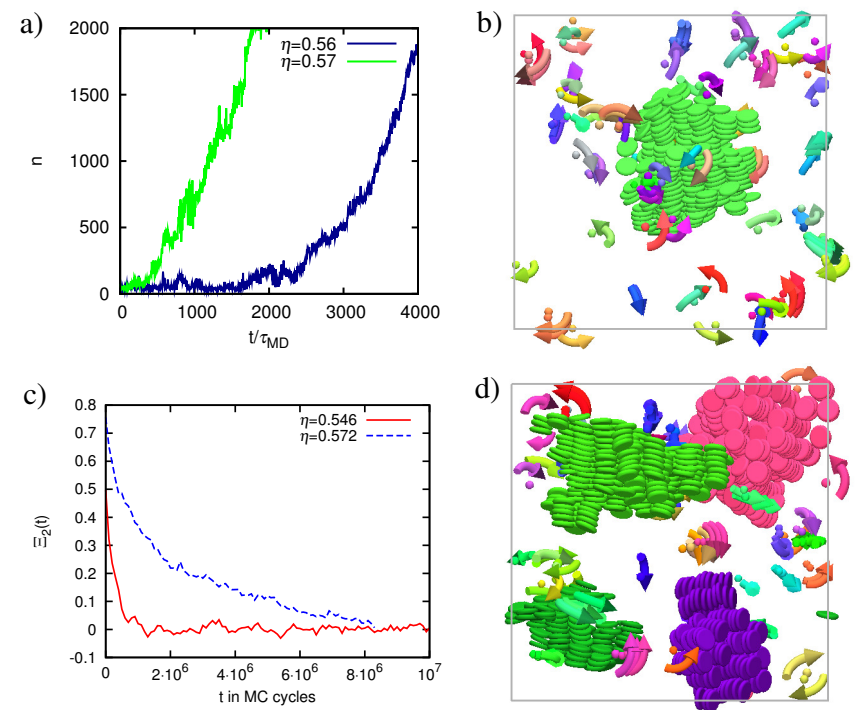

d)

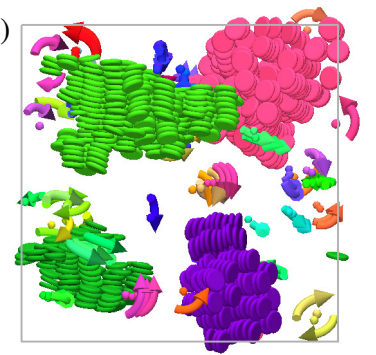

FIG. 2. (Color online) (a) The size of the largest columnar cluster $n$ in an MD simulation of an isotropic fluid of $N=10000$ OHSC with $L / D=0.2$ at packing fraction $\eta=0.56$ and $\eta=0.57$. (b) and (d) Typical configurations of the largest columnar clusters, when the size of the largest cluster is around 300 particles (post-critical) for $\eta=0.56$ (b) and $\eta=0.57$ (d). Different colors of the particles denote different orientations. Swiftly rotating fluid particles are denoted by arrows pointing into the direction of the rotation; the other fluid particles are not shown at all. (c) The orientational correlation function $\Xi_{2}(t)$ as a function of MC cycles for $\eta=0.546$ and 0.572 .

for particles with sharper edges.

We first consider a suspension of $N$ OHSC with aspect ratio $L / D=0.2$ in a volume $V$ or at a pressure $P$. This system displays a bulk transition from an isotropic phase with packing fraction $\eta_{I} \equiv v_{\mathrm{OHSC}} N / V=0.5050$ to a columnar phase with $\eta_{C}=0.5691$ at pressure $P^{*}=\beta P v_{\mathrm{OHSC}}=8.27$, where $\beta=1 / k_{B} T$ and $v_{\mathrm{OHSC}}$ is the volume of the OHSC particle [5].

In order to study the spontaneous formation of the columnar phase from the isotropic fluid phase, we require a cluster criterion that enables us to identify the columnar clusters. Unfortunately, the cluster criterion that was introduced to study nucleation of the nematic, smectic, and crystal phase in systems of colloidal hard rods [9 11, is not strict enough to identify columnar clusters. We therefore developed a new cluster criterion that enables us to detect columnar clusters. Particles are considered eligible for inclusion into a columnar cluster if they have sufficient neighbors with columnar order. Neighbors are considered to have columnar order when they have sufficient hexagonal order, as measured by a standard order parameter, but do not show high ordering with another symmetry. See 12 for details.

We use event driven Molecular Dynamics (MD) simulations of relatively large system sizes $(N=1500,3000$, and 10000) to study the kinetics of the IC phase transfor-
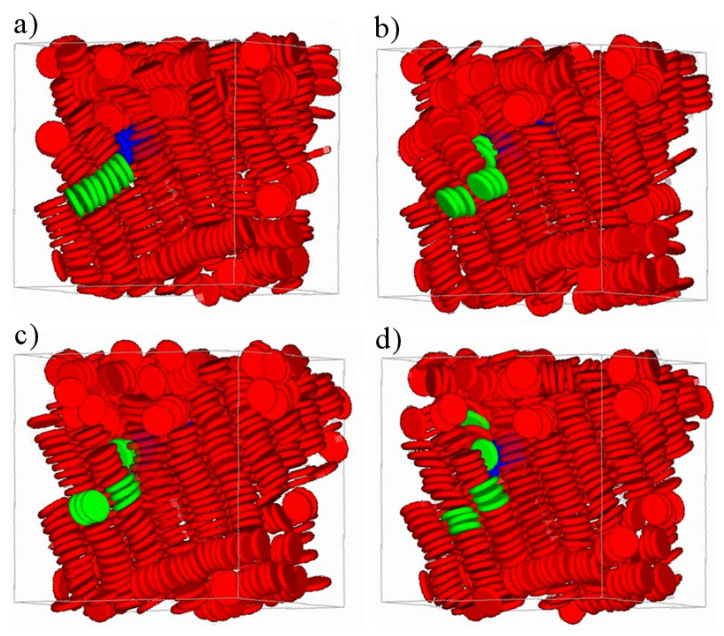

FIG. 3. (a) A long stack of (green) particles, which is oriented perpendicular to the nematic director of the columnar phase, is divided in smaller packages of two to four particles as shown in (b). Subsequently, these smaller packages of particles can rotate by 90 degrees as shown in (c) and (d) in such a way that the orientation matches the columnar phase.

mation. Time is measured in units of $\tau_{\mathrm{MD}}=\sqrt{\beta m D^{2}}$, where $m$ is the mass of an OHSC. For $\eta \geq 0.56$, we observe the immediate formation of short stacks of OHSC in the supersaturated isotropic fluid phase, which subsequently tend to orient perpendicular to each other to optimize the packing. The symmetry of these locally favored structures in the resulting cubatic phase is incompatible with that of the columnar phase, and hence the cubatic orientational order can be seen as geometric frustration against the formation of the columnar phase. The cubatic order is more pronounced and longer ranged for higher $\eta$ and smaller system sizes $(N=1500$ and 3000). A typical configuration of such a cubatic phase is shown in Fig. 1(b) for $\eta=0.57$. However, in very long simulations the cubatic phase always transforms into a columnar phase. In order to analyze the phase transformation, we show the time evolution of the largest columnar cluster identified by our cluster criterion in Fig. $2 \mathrm{a}$ for $\eta=0.56$ and 0.57 . We clearly observe that the cluster grows much slower for lower $\eta$ due to the lower supersaturation. In addition, we present typical configurations of post-critical columnar clusters in Fig. $2 \mathrm{~b}$ and d. At a packing fraction of $\eta=0.56$, we observe one columnar cluster that grows further to form the stable columnar phase, while for $\eta=0.57$ three post-critical clusters are observed as the nucleation barrier is much lower. Interestingly, the nematic directors of the columnar clusters are aligned along the three preferred axes of the cubatic phase, where it originated from, see Fig. 2 $\mathrm{d}$. We conclude that the IC phase transformation proceeds via a transient cubatic phase and corresponds to a nucleation and growth scenario in which a spontaneously formed columnar cluster grows out to form the stable columnar phase. During MD simulations with $N=10000$, we 
observe the appearance of columnar clusters before longrange cubatic order appeared after quick compression. In fact, this was to be expected, as the time it takes for the cubatic order to spread throughout the system increases with system size, while the time for a nucleus to form in a fixed volume is system size independent. Therefore, the behavior observed in experiments, such as the Cryo-TEM experiments in which the cubatic phase was observed [3, is largely dependent on the sample volume, which for Cryo-TEM is rather small to allow sufficiently fast shock-freezing of the sample. This suggest that the cubatic phase may be stabilized by confinement.

Additionally, heterogeneous dynamics in the form of collective particle reorientations is found in the locally cubatic fluid, as can be appreciated from Fig. $2 \mathrm{~b}$ and d, where we represent all fluid particles that rotate swiftly by arrows denoting the direction of the rotation. We wish to remark here that the clusters of particles which rotate simultaneously can be easily identified here as small stacks of up to four particles, which is impossible for glassy states of spherical particles where heterogeneous dynamics cannot be easily related to the local structure [13. Similarly, the growth of a columnar phase often proceeds by collective attachment of small stacks rather than single particles [12. Interestingly, the rotation of stacks also plays a crucial role in the late-stage development of the columnar cluster. Fig. 3 shows a form of defect-healing in which a stack of mis-aligned particles in the columnar cluster first breaks up into smaller stacks and, subsequently, these smaller stacks reorient to conform with the director of the cluster.

As nucleation of a columnar phase from a glassy state with cubatic order is hardly studied, it is interesting to determine the nucleation barrier associated for this devitrification process. Since the equilibration can only proceed via collective rearrangement of small clusters, the formation of the columnar phase is severely hampered by slow dynamics. We determine the nucleation barrier by employing the umbrella sampling technique in Monte Carlo (MC) simulations 9. To obtain an estimate of the number of MC cycles needed to sample the configurational space sufficiently, we study first the translational and orientational dynamics of the particles in the cubatic phase. To this end, we calculate the mean square displacement $\left\langle\left(\mathbf{r}_{i}(t)-\mathbf{r}_{i}(0)\right)^{2}\right\rangle$ (not shown) and the orientational correlation function $\Xi_{2}(t)=$ $\left\langle\frac{3}{2}\left[\mathbf{u}_{i}(t) \cdot \mathbf{u}_{i}(0)\right]^{2}-\frac{1}{2}\right\rangle$, where the angular brackets indicate an ensemble average and $t$ indicates the time measured in MC cycles. Exemplarily, Fig. 2b displays $\Xi_{2}(t)$ for $\eta=0.546$ and 0.572 . Similar behavior is seem in the event-driven MD simulations [12, where a clear slowing down of the translational and rotational dynamics by about a factor of $\simeq 6$ is observed when the packing fraction is increased from $\eta=0.5$ to 0.56 . The simultaneous slowing down of the translational and rotational dynamics should be contrasted with the decoupling of the freezing of the translational and rotational degrees of freedom that is found for ellipsoids [14, 15].

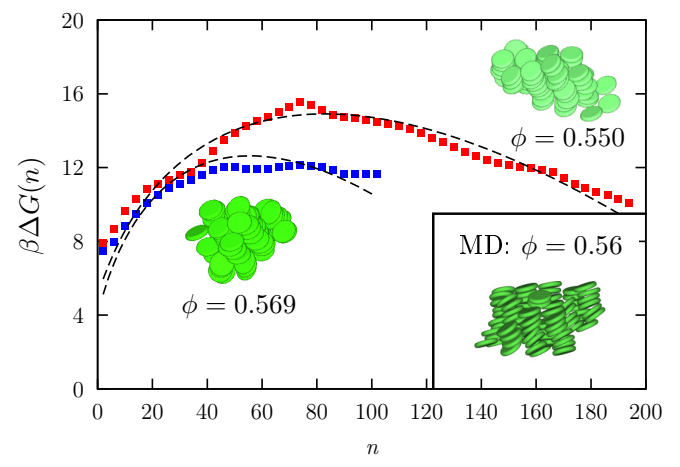

FIG. 4. Gibbs free energy $\beta \Delta G(n)$ as a function of the number of particles $n$ in the largest columnar cluster for a system of OHSC with $L / D=0.2$ and packing fraction $\eta=0.550$ and 0.569 (squares). The dashed line is a fit [16 to classical nucleation theory. Configurations of the critical nucleus are shown obtained from MC for $\eta=0.550$ and 0.569 in the main panel and from MD simulations at $\eta=0.56$ in the inset.

Subsequently, we determine the Gibbs free energy $\Delta G(n)$ as a function of columnar cluster size $n$ using the umbrella sampling technique [9]. The nucleation barriers for OHSC with $L / D=0.2$ and $\eta=0.550$ and 0.569 are shown in Fig. 4. As equilibration is rather slow due to glassy dynamics, the noise on $\Delta G(n)$ is significant. However, we are able to determine for the first time a nucleation barrier for a devitrification process in which a glassy state transforms into a stable phase via collective particle reorientations. We also present typical configurations of the critical nucleus that corresponds with the top of the barrier in Fig. 4 along with the critical nucleus obtained from MD simulations at $\eta=0.56$. We find that the results for the structure and shape of the critical nucleus as obtained from MC or MD simulations are very similar, i.e., the cluster consists of an hexagonal array of particle stacks and the overall shape of the cluster is roughly spherical. The barrier height $\beta \Delta G^{*}(n) \simeq 15$ and 12 for $\eta=0.550$ and 0.569 , respectively. We mention here that the corresponding values for the supersaturation $\beta \Delta \mu=0.414$ and 0.553 , which is the driving force for nucleation, is extremely small, for comparison we note that, for hard spheres at $\eta=0.5478, \beta \Delta \mu \simeq 0.74$, which leads to fast nucleation [16].

Our results show clearly that the cubatic phase of OHSC is metastable with respect to an IC phase transition. In this light, it is interesting to study the effect of particle shape on the stability of the cubatic phase. To this end, we measure the cubatic order parameter [7] as a function of pressure for the three particle shapes depicted in Fig. 1 using NPT MC simulations with $N=3000$ particles. The shapes (ordered from more curved to more cylinder-like) are OHSC, HCS, which resemble recently synthesized particles of Ref. [18], and the double hard cut spheres (DHCS). The latter model consists of two superimposed HCS and is essentially a cylinder. All three models have the same height-to-diameter ratio of $L / D=0.2$. 


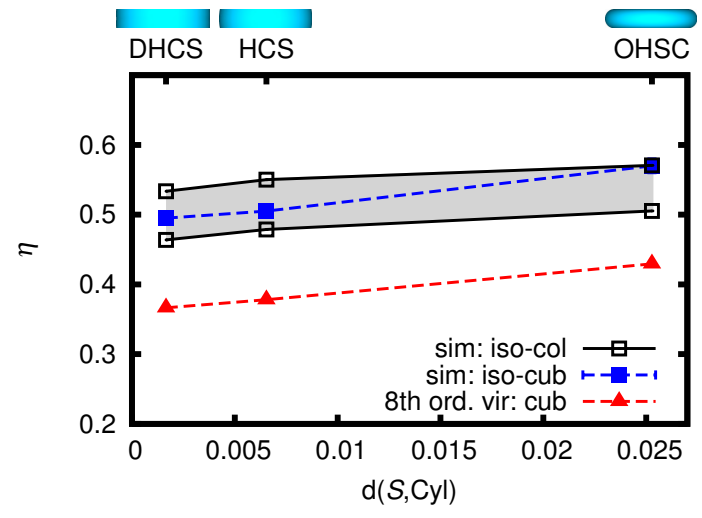

FIG. 5. The phase diagram for double hard cut spheres (DHCS), hard cut spheres (HCS) and OHSC with $L / D=$ 0.2 : packing fraction $\eta$ versus $d(S, \mathrm{Cyl})$, the difference [12, 17. between the shape $S$ in question and a cylinder (Cyl) with the same volume and aspect ratio. The gray area and the black lines denote coexistence between the isotropic and the columnar phase from simulations 12. The metastable transition from the isotropic to the cubatic phase is denoted by the dashed lines, where the solid squares denote simulation results for $N=3000$ particles, and the triangles denote the results from 8 th order virial theory.

The onset of cubatic order, as defined by the packing fraction at which the cubatic order parameter suddenly increases, is shown as the dashed, blue line in Fig. 5. We observe clearly that the cubatic order sets in at lower $\eta$ upon decreasing the particle curvature.

In addition, we study the stability of the cubatic phase using a high-order virial theory. In Ref. [7, it was shown that a 8th-order expansion is required to predict a stable isotropic-cubatic phase transition for HCS with $L / D=0.2$. Here, we apply this theory to predict the isotropic-cubatic phase transition for OHSC, HCS (with higher precision than in Ref. [7), and DHCS. The results for the bulk density of the cubatic phase in coexistence with the isotropic phase are denoted by the dashed, red lines in Fig. 5k at the 8th virial level. Although, the agreeement with the simulations results is not satisfactory, surprisingly, the trend of the bulk density of the cubatic phase with particle curvature is very similar to that of the NPT MC simulations.

Finally, we determine the stability of the cubatic phase with respect to the columnar phase for HCS for which this phase was originally discovered [6]. The apparent stability of the cubatic phase for HCS could be due to dynamic arrest. Inspired by the particle stack rotations as observed in our MD simulations, we introduce a new cluster move in the $\mathrm{MC}$ simulations to speed up equilibration 12. In order to investigate more precisely whether or not there is phase coexistence between an isotropic fluid phase with either a cubatic phase or a columnar phase, we perform NPT MC simulations of the two coexisting phases in a simulation box that is large enough that the interfacial free energy is sufficiently small [19, 20]. The phase that grows at the expense of the other phase must be the stable phase. The pressure at coexistence can be determined as the pressure at which the growth speed of the columnar phase is zero. The corresponding coexistence densities for HCS and DHCS are shown in Fig. 5 . Clearly, the pressure at which the columnar phase becomes more stable than the isotropic phase is lower than the pressure at which long-range cubatic order was found, which unambiguously shows that the cubatic phase is unstable for all three shapes considered.

In conclusion, we find that the cubatic phase is metastable with respect to an IC phase coexistence for all three model platelets, and can be regarded as a transient phase in the IC phase transformation. The locally favored structures of perpendicularly oriented particle stacks in the cubatic phase leads to geometric frustration that prevents the formation of the columnar phase thereby yielding vitrification. Additionally, we find a direct link between structural order and dynamic heterogeneities provided by the cooperative rotation of particle stacks in the cubatic phase. Such a link is often assumed to be characteristic for glassy behavior, but is not easy to demonstrate in e.g., colloidal hard sphere glasses. We also show that the cooperative stack rotations play an important role in the devitrification process and that the life time of the cubatic phase can be tuned by confinement and by surprisingly small differences in the particle shape. Interestingly, our results explain recent experimental observations on suspensions of gibbsite platelets which enter a kinetically arrested glass regime upon increasing the particle concentration and in which small iridescent grains of the columnar phase were formed after periods of months to years [4].
[1] H. Shintani and H. Tanaka, Nat. Phys. 2, 200 (2006).

[2] F. Frank, Proc. Phys. Soc. London, Sec. A 215, 43 (1952).

[3] S. J. S. Qazi, G. Karlsson, and A. R. Rennie, J. Colloid Interf. Sci. 348, 80 (2010).

[4] M. C. D. Mourad, A. A. Verhoeff, D. V. Byelov, A. V. Petukhov, and H. N. W. Lekkerkerker, J. Phys. Cond. Matt. 21, 474218 (2009).

[5] M. Marechal, A. Cuetos, B. Martínez-Haya, and M. Dijkstra, J. Chem. Phys. 134, 094501 (2011).
[6] J. A. C. Veerman and D. Frenkel, Phys. Rev. A 45, 5632 (1992).

[7] P. D. Duncan, M. Dennison, A. J. Masters, and M. R. Wilson, Phys. Rev. E 79, 031702 (2009).

[8] P. D. Duncan, A. J. Masters, and M. R. Wilson, Phys. Rev. E 84, 011702 (2011).

[9] A. Cuetos and M. Dijkstra, Phys. Rev. Lett. 98, 095701 (2007).

[10] A. Patti and M. Dijkstra, Phys. Rev. Lett. 102, 128301 (2009). 
[11] R. Ni, S. Belli, R. van Roij, and M. Dijkstra, Phys. Rev. Lett. 105, 088302 (2010).

[12] See supplementary material at http://link.aps.org/ supplemental for movies and technical details.

[13] E. Sanz, C. Valeriani, E. Zaccarelli, W. C. K. Poon, P. N. Pusey, and M. E. Cates, Phys. Rev. Lett. 106, 215701 (2011).

[14] C. De Michele, R. Schilling, and F. Sciortino, Phys. Rev. Lett. 98, 265702 (2007).

[15] M. Letz, R. Schilling, and A. Latz, Phys. Rev. E 62,
$5173(2000)$.

[16] L. Filion, M. Hermes, R. Ni, and M. Dijkstra, J. Chem. Phys. 133, 244115 (2010).

[17] M. Moszyńska, Selected topics in convex geometry (Birkhäuser, Boston, 2006).

[18] T. Fujibayashi and M. Okubo, Langmuir 23, 7958 (2007).

[19] C. Vega, E. P. A. Paras, and P. A. Monson, J. Chem. Phys. 96, 9060 (1992).

[20] T. Zykova-Timan, J. Horbach, and K. Binder, J. Chem. Phys. 133, 014705 (2010). 